\title{
Réalisez-vous le plein potentiel de la pratique de la pharmacie dans le système de santé?
}

\author{
par Lauza Saulnier
}

$\mathrm{D}$ ans le milieu contemporain de la santé complexe et trépidant, les leaders en pharmacie doivent assurer l'utilisation des ressources optimale à la prestation de services de première qualité, efficaces et sécuritaires. Les équipes de pharmacie hautement efficaces travaillent sans relâche à concevoir et mettre en œuvre des programmes et des services en vue d'améliorer les résultats cliniques et de faire évoluer les pratiques d'utilisation sécuritaire des médicaments.

Mais, notre profession a-t-elle maximisé son apport au système de santé? Quel progrès avons-nous réalisé pour élargir le champ de pratique? Quelles stratégies avons-nous déployées afin d'accorder la priorité aux activités de la pratique qui auraient le plus d'influence sur les soins aux patients?

Surveiller les indicateurs clés de rendement est une stratégie nécessaire pour évaluer la qualité de l'exercice de la pharmacie clinique et pour soutenir la transparence et la responsabilisation professionnelle. Malheureusement, une telle surveillance demeure mal intégrée dans bon nombre de programmes de pharmacie hospitalière partout au pays. Au cours des dernières années, plusieurs initiatives ont été mises en place afin de favoriser les pratiques de gestion des médicaments s'appuyant sur des données probantes et de faciliter l'évaluation du rendement.

Les indicateurs clés de rendement relatifs à la pharmacie clinique (ICRpc) au Canada ont été conçus grâce à un processus d'établissement de consensus dans le but de faire avancer la pratique de la pharmacie clinique pour ainsi améliorer la qualité des soins et les résultats thérapeutiques (consultez le https://cshp. ca/clinical-pharmacy-key-performance-indicators). L'ensemble fondamental des ICRpc fondés sur des données probantes permet aux pharmaciens qui travaillent dans les milieux hospitaliers de soins de courte durée de concentrer leurs efforts sur les principales interventions cliniques; il offre aussi une méthode structurée pour mesurer la qualité des soins directs aux patients et de la prestation des services.

Le document Pratique de la pharmacie dans les hôpitaux et les autres milieux de soins collaboratifs : déclarations de principes (disponible au https://cshp.ca/position-statements) présente la prise de position de la Société canadienne des pharmaciens d'hôpitaux (SCPH) et décrit le niveau de performance souhaité et réalisable qu'on peut attendre de la pratique de la pharmacie. Cet ensemble

de déclarations de principes sert de fondement au programme Excellence en pharmacie hospitalière de la SCPH. Ce dernier est conçu pour aider les membres à s'appliquer à l'amélioration des résultats thérapeutiques grâce aux soins centrés sur le patient, aux meilleures pratiques, et à la communication et à la collaboration. Quinze indicateurs de rendement ont été choisis pour mesurer les progrès réalisés vers l'excellence dans la pratique de la pharmacie (https://www.cshp.ca/sites/default/files/Excellence/ ExcellenceFlyer_Revised.pdf).

Depuis plus de 30 ans, le Rapport sur les pharmacies hospitalières canadiennes représente l'une des principales références et un outil d'étalonnage de premier choix pour les services de pharmacie hospitalière partout au Canada et dans le monde. En 2017, le conseil de la SCPH a accepté la demande du comité de rédaction $\mathrm{du}$ Rapport sur les pharmacies hospitalières canadiennes qui souhaitait devenir l'un des conseils affiliés de la Société. Le conseil de la SCPH chargé du sondage sur les pharmacies hospitalières canadiennes, comme il se nomme maintenant, a réalisé son sondage de 2016-2017 sous l'égide de la SCPH. Les résultats ont été publiés récemment au http://www.lillyhospitalsurvey.ca/hpc2/ content/ReportsF3.asp .

Des mesures du rendement publiées dans le Rapport sur les pharmacies hospitalières canadiennes et celles comprises dans le programme Excellence en pharmacie hospitalière aident à suivre les progrès relatifs à l'atteinte des objectifs, à comparer le rendement à des étalons, à évaluer la valeur réelle des programmes et services, et à repérer les possibilités d'amélioration. Les mesures du rendement sont des éléments essentiels à l'incitation au changement dans une quête continuelle de l'excellence en pratique professionnelle.

La SCPH est depuis longtemps reconnue pour faire évoluer la pratique de la pharmacie dans les hôpitaux et les autres milieux de soins de santé misant sur la collaboration. Maintenant, défions-nous d'obtenir les meilleurs résultats thérapeutiques pour nos patients!

[Traduction par l'éditeur]

Lauza Saulnier, B. Sc. (Pharm.), A.C.P.R., est présidente sortante et agente de liaison pour la vision de la Société canadienne des pharmaciens d'hôpitaux. 